\title{
Indoor Residual Clutter Characterization for UWB Sensor Radar Networks
}

\author{
Flavio Morselli, ${ }^{*}$ Stefania Bartoletti, ${ }^{\dagger}$ and Andrea Conti* \\ *DE, CNIT at University of Ferrara, Ferrara, Italy, E-mail: flavio.morselli@unife.it, a.conti@ieee.org \\ ${ }^{\dagger}$ IEIIT, CNIT at National Research Council, Bologna, Italy, E-mail: stefania.bartoletti@cnr.it
}

\begin{abstract}
Sensor radar networks (SRNs) employing ultrawideband (UWB) signals are a prominent solution for accurate localization and tracking in indoor environments. However, tracking device-free targets via SRNs is challenging, especially in environments heavily affected by clutter. Clutter characterization is vital to derive performance benchmarks as well as to design inference algorithms for SRNs. Examples of clutter statistical characterization have been provided in the literature for conventional SRNs employing narrowband signals in outdoor scenarios. However, considerably less effort has been devoted for SRNs employing UWB signals in indoor environments. This paper proposes an approach to characterize the clutter-plus-noise component after mitigation filtering in UWB SRNs. In particular, the statistical properties of the residual clutter-plus-noise are derived by applying statistical tests on measurements gathered in an indoor environment via UWB sensor radar networks.
\end{abstract}

Index terms - Sensor radar network, clutter, UWB, tracking, network experimentation.

\section{INTRODUCTION}

Tracking device-free people or objects via sensor radar networks (SRNs) is a key enabler for important applications, including smart cities [1], autonomous vehicles [2], and public safety [3]. Accurate modeling of the wireless impairments affecting the SRNs' operations is essential to design SRNs capable of achieving satisfactory performance in terms of localization and tracking accuracy [4]-[8]. Among various wireless impairments, the clutter, i.e., the unwanted echoes generated by scatterers not meaningful for the target localization process, represents one of the major challenges [9].

The clutter, if not mitigated or properly taken into account, might lead to noticeable performance degradation [10]. In particular, an accurate characterization of the clutter is essential to establish performance benchmarks, develop robust mitigation filters, and design inference algorithms [11]-[14]. However, clutter characterization is a difficult task due to the heterogeneous operation conditions encountered in real applications. The ability to distinguish between clutter and useful echoes (i.e., echoes that carry positional information about the targets) depends on the specific environment and application. For example, in synthetic-aperture radars the echoes determined by the background are essential to obtain an

This research was supported, in part, by the Office of Naval Research Global under Grant N62909-18-1-2017 and by the European Union's Horizon 2020 research and innovation programme under Grant 871249 . accurate image of the environment [15], while in surveillance radar they deteriorate the detection capability [16]. In general, the characteristics of the echoes depend on: (i) the environment (e.g., outdoor, sea, or indoor); (ii) SRN operation (e.g., monostatic vs. multistatic); and (iii) the signaling employed (e.g., continuous wave or impulsive).

The clutter can be modeled as a stochastic process, whose characteristics vary if observed prior or after clutter mitigation filtering. Several statistical models have been proposed in the literature to describe the clutter amplitude or power and its variability in terms of Doppler spectrum [17]-[21]. Despite clutter characterization has been largely studied for narrowband radars in a variety of frequency bands and operating conditions, no well-established counterparts exist for SRNs operating in indoor environments with wideband and ultrawideband (UWB) signals. Recently, this type of SRNs is conveying a lot of interest for tracking device-free objects in indoor environments thanks to the ranging accuracy, multipath resolution, and penetration characteristics provided by UWB signals [22]-[26]. The performance analysis (e.g., the derivation of theoretical bounds) and the design of algorithms (e.g. coherent or noncoherent detectors for ranging) for UWB SRNs rely on the statistics of the clutter-plus-noise component after clutter mitigation filtering. In particular, the clutter-plusnoise distribution and its statistical properties in terms of stationarity and correlation greatly affect the design of the inference algorithms [27].

In indoor environments, the echoes due to the background and static objects (static clutter) are typically greater in amplitude and exhibit a longer duration compared to the ones originated by moving scatterers (dynamic clutter). Therefore, the characterization and mitigation of the clutter component generated by static scatterers is crucial for UWB SRNs. The goal of this paper is to propose an approach for the statistical characterization of the residual clutter-plus-noise after and to show its application on a real dataset of measurements taken with a UWB SRNs in an indoor environment. We first collect the dataset of measurements with a monostatic UWB SRN in the presence of only static scatterers. Then, the measurements are processed with different clutter mitigation filters, namely, empty room (ER) [4], exponential averaging filter (EAF) [28], and single-delay canceller (SDC) [9]. Finally, the stationarity, correlation, and distribution of the sample amplitudes are 
assessed based on the waveforms at the filter's output. More specifically, the clutter is considered as a Gaussian process, where the correlation is tested using the Ljung-Box test (LBT) [29] and the Gaussianity is tested using the Anderson-Darling test (ADT) [30]. The key contributions of the paper can be summarized as follows:

- characterization of the residual clutter-plus-noise component in terms of a stochastic process in an indoor environment; and

- derivation of the main statistical properties based on statistical tests applied on collected measurements.

Notations: A random variable (RV) and its realization are denoted by $\mathrm{x}$ and $x$; a continuous stochastic process and its sample function are denoted by $\mathrm{x}(t)$ and $x(t) ; f_{\mathrm{x}}(x)$ denotes the probability density function (PDF) of a continuous RV $\mathrm{x}$; $F_{\mathrm{x}}(x)$ denotes the cumulative distribution function (CDF) of a continuous $\mathrm{RV} \times$; $\lfloor x\rfloor$ denotes the greater integer less then or equal to $x ; \ln (x)$ denotes the natural logarithm of $x$; and $|\mathcal{A}|$ denotes the cardinality of the set $\mathcal{A}$.

\section{System Model \& EXPERIMENTATION}

This section presents the system model and describes the received signal as well as the clutter mitigation filters considered. Then, the measurement campaign is described, on which the statistical characterization is based.

\section{A. System Model}

Consider a SRN in a monostatic or multistatic configuration (i.e., where the transmitters and the receivers are colocated or separated, respectively) with sensor using UWB impulse-radio technologies [31]. The transmitter emits an UWB impulse $u(t)$ at time instant $j T_{\mathrm{f}}$ where $j \in \mathbb{Z}$ and $T_{\mathrm{f}}$ is the frame time. The impulse propagates in the environment and is backscattered by targets and other scatterers (static or dynamic) that are present in the monitored area. The receivers observe the backscattered echoes within a fixed observation time $T_{\mathrm{o}} \leq T_{\mathrm{f}}$ before the next impulse is transmitted. The received signal $r(t)$ at the generic sensor radar (SR) receiver can be written as the superposition of the backscattered impulses transmitted at time $j T_{\mathrm{f}}$. Specifically,

$$
r(t)=\sum_{j=-\infty}^{+\infty} r^{(j)}(t)
$$

where $r^{(j)}(t)$ is the signal received in the $j$-th frame, which is given by

$$
\mathbf{r}^{(j)}(t)=\mathbf{s}^{(j)}(t)+\mathrm{c}^{(j)}(t)+\mathrm{w}^{(j)}(t)
$$

for $t \in\left(j T_{\mathrm{f}}, j T_{\mathrm{f}}+T_{\mathrm{o}}\right]$ and 0 elsewhere. In (2), the process $\mathbf{s}^{(j)}(t)$ encapsulates the informative components for the localization process and contains the signal backscattered from the targets as well as the undesired multipath propagation involving reflections on the targets. Due to the targets' mobility, its statistical properties vary from frame to frame. The process $\mathrm{c}^{(j)}(t)$ represents the undesired clutter components due to dynamic and static scatterers whose statistical properties vary between frames due to the mobility of dynamic scatterers. Notice that the processes $\mathbf{s}^{(j)}(t)$ and $\mathbf{c}^{(j)}(t)$ are not independent. The targets' motion affects the signals backscattered from both static and dynamic scatterers, while the motion of the dynamic scatterers affects the targets' multipath. Last, the process $w^{(j)}(t)$ is a zero-mean additive white Gaussian noise (AWGN) representing the thermal noise component whose statistical properties do not vary between frames.

Due to the fine resolution provided by the UWB signals, single scatterers cannot be considered point reflectors as in narrowband SRNs and should be treated as range-spread objects [15]. In this case, reflections are made up by infinitesimal contributes determined by the illuminated objects surface. Then, the targets and clutter process cannot be written as a finite sum of discrete components and in the $j$-th frame interval the components related to the targets backscatter is [32]

$$
\mathbf{s}^{(j)}(t)=\int_{0}^{T_{\mathrm{o}}} u(t-\tau) \boldsymbol{\varsigma}^{(j)}(\tau) d \tau
$$

where $\boldsymbol{\varsigma}^{(j)}(\tau)$ is a stochastic process representing the distributed reflections from the targets. Similarly, the components related to the clutter is

$$
\mathrm{c}^{(j)}(t)=\int_{0}^{T_{\mathrm{o}}} u(t-\tau) \mathbf{\kappa}^{(j)}(\tau) d \tau
$$

where $\mathbf{k}^{(j)}(\tau)$ is a stochastic processes representing the distributed reflections from the dynamic and static scatterers.

Various clutter mitigation filters are available in literature [4], [9], [28]. In particular, we will focus on the ER, EAF, and SDC, all of which aim to the estimate the clutter response and subtract it from the received frames. In the case of ER, the filtered signal after clutter mitigation can be written as

$$
x^{(j)}(t)=r^{(j)}(t)-\hat{c}(t)
$$

where $\hat{c}(t)=\sum_{h=1}^{N_{\mathrm{a}}} r^{(h)}(t) / N_{\mathrm{a}}$ is an estimate of the response of the wireless environment in absence of targets, i.e., only clutter. This technique exhibits good performance in static environments and is suitable for implementation on low complexity devices. The main drawback is represented by the poor performance in quasi-static or dynamic environments and its non adaptive nature. The EAF overcomes some of the ER downsides by iteratively updating the environment response. The filtered signal is given by

$$
\mathrm{x}^{(j)}(t)=\mathrm{r}^{(j)}(t)-\hat{\mathrm{c}}^{(j)}(t)
$$

where the clutter response is updated frame by frame as $\hat{\mathbf{c}}^{(j)}(t)=\beta \hat{\mathbf{c}}^{(j-1)}(t)+(1-\beta) \mathbf{r}^{(j)}(t)$. The parameter $\beta \in(0,1)$ weights the clutter signal at the previous frame and the current received frame to obtain the current clutter estimate. This mitigation filter rejects the static clutter while being robust to slow environmental changes. However, echoes originated by slow moving targets are also attenuated, potentially causing performance degradation. On the other hand, the SDC filter estimates the clutter response as the previous received frame, 
TABLE I

SR AND MEASUREMENT CAMPAIGN PARAMETERS.

\begin{tabular}{|l|l|}
\hline Parameter (Symbol) & Value \\
\hline SR pulse shape & Gaussian \\
SR carrier frequency & $7.26 \mathrm{GHz}$ \\
SR pulse bandwidth & $1.4 \mathrm{GHz}$ \\
SR energy per pulse & $2.6 \mathrm{pJ}$ \\
Repetition interval $\left(T_{\mathrm{f}}\right)$ & $40 \mathrm{~ms}$ \\
Observation time $\left(T_{\mathrm{o}}\right)$ & $43.4 \mathrm{~ns}$ \\
Sample time $\left(T_{\mathrm{s}}\right)$ & $42.8 \mathrm{ps}$ \\
Number of configurations tested & 12 \\
Number of frames recorded per configuration $(M)$ & 250 \\
Number of samples per frame $(N)$ & 1245 \\
\hline
\end{tabular}

i.e. $\hat{c}^{(j)}(t)=r^{(j-1)}(t)$, and the filtered signal can be written as

$$
x^{(j)}(t)=r^{(j)}(t)-r^{(j-1)}(t) .
$$

The SDC is particularly suitable for high maneuvering targets and exhibits good performance even for dynamic environments, until the per-frame variability of the targets echoes is greater than the clutter one.

\section{B. Static Clutter}

Regardless of the clutter mitigation filter employed, from (2) the filtered received signal can be rewritten as

$$
\mathbf{x}^{(j)}(t)=\tilde{\mathbf{s}}^{(j)}(t)+\mathbf{n}^{(j)}(t)
$$

where $\tilde{\mathbf{s}}^{(j)}(t)$ represents the filter output when the input is $\mathbf{s}^{(j)}(t)$ and $\mathbf{n}^{(j)}(t)$ represents the residual clutter-plus-noise, which is the filter output when the input is $\mathrm{c}^{(j)}(t)+\mathrm{w}^{(j)}(t)$. A statistical characterization of $n^{(j)}(t)$ is required to design algorithms for detecting $\tilde{\mathbf{s}}^{(j)}(t)$ or inferring parameters related to $\tilde{\mathbf{s}}^{(j)}(t)$. Furthermore, the theoretical analysis of such algorithms (e.g. derivation of the detection probability, false alarm probability, estimation error, and variance) depends on the underlying statistical model of the signals [25], [27], [32].

In general, a complete characterization of the process $\mathbf{n}^{(j)}(t)$ based on the filtered signal $x^{(j)}(t)$ is difficult due to the time-varying nature of the process and the interdependency between the clutter-plus-noise and targets processes. However, we can initially consider only the clutter determined by static scatterers (e.g., ground, walls, and furnitures) in the absence of targets. In such a scenario, the filtered signal in (8) simplifies to $x^{(j)}(t)=\mathrm{n}^{(j)}(t)$, where only the residual clutter-plus-noise is observed at the filter's output. The statistical properties of $\mathrm{n}^{(j)}(t)$ do not depend on the particular frame index $j$. Therefore, the process $x^{(j)}(t)$ can be considered as a time shift of an underlying process $\mathrm{x}(t)$. A set of $M$ sample functions $\left\{x^{(j)}(t)\right\}_{j \in \mathcal{M}}$ with frame index set $\mathcal{M}=\{1,2, \ldots, M\}$ can be regarded as different realizations of the process $x(t)$. This allows to determine the statistical properties of $x(t)$ based on successive received frames $\left\{x^{(j)}(t)\right\}_{j \in \mathcal{M} \text {. }}$

Remark: The echoes due to the background and large static objects are typically greater in amplitude and exhibit a longer duration compared to the ones originated by moving scatterers. Moreover, the absence of dynamic scatterers allows to consider

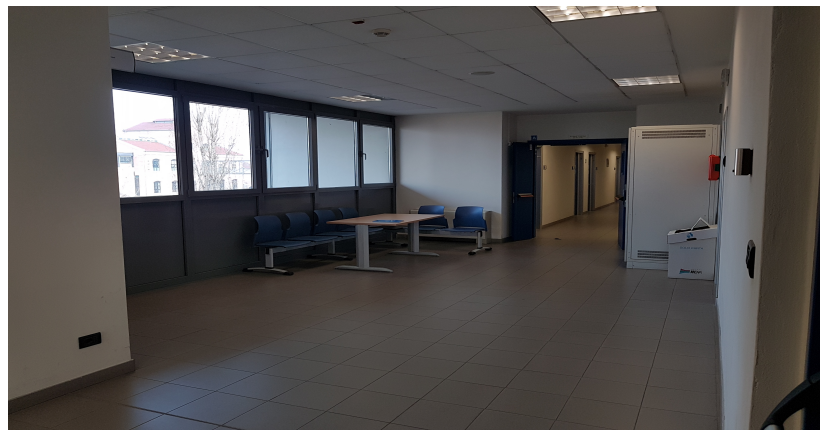

Fig. 1. Measurement campaign environment in the Department of Engineering at the University of Ferrara.

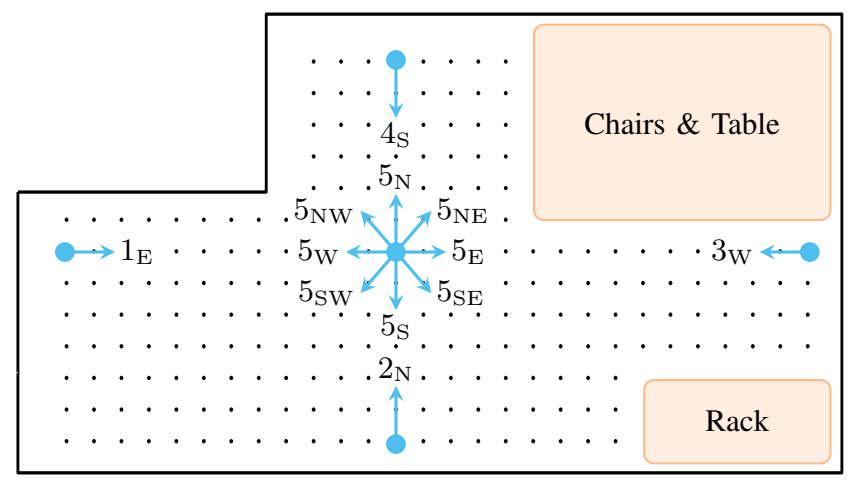

Fig. 2. Map of the environment with the different SR spatial configurations. The spacing between the grid points is approximatively $0.3 \mathrm{~m}$.

approximatively independent the processes $\tilde{\mathbf{s}}^{(j)}(t)$ and $\mathbf{n}^{(j)}(t)$, with the targets' motion determining only partial shadowing of the static reflectors. Therefore, the clutter characterization in a scenario with no targets and only static scatterers is crucial for addressing the clutter characterization problem.

\section{Measurement Campaign}

The clutter characterization will be based on measurements obtained with a monostatic UWB SR in an indoor environment. Then, we define the discrete version of $\mathrm{x}(t)$ as $\mathrm{x}_{i}=\mathrm{x}\left(i T_{\mathrm{s}}\right)$, where $i \in \mathcal{N}=\{1,2, \ldots, N\}, \mathcal{N}$ is the discrete index set in which $N=\left\lfloor T_{\mathrm{o}} / T_{\mathrm{S}}\right\rfloor$ is total number of sample in an observation interval, and $T_{\mathrm{s}}$ is the sampling time. Denote with $x_{i}^{(j)}$ the $i$-th sample of the $j$-th realization $x^{(j)}(t)$, i.e. $x_{i}^{(j)}=x^{(j)}\left(i T_{\mathrm{s}}\right)$. The statistical characterization is based on the samples set $\left\{x_{i}^{(j)}\right\}_{i \in \mathcal{N}}^{j \in \mathcal{M}}$.

The measurements were carried out within an area of approximatively $9 \mathrm{~m} \times 4 \mathrm{~m}$, where the presence of a table, chairs, panels, walls, and other objects leads to a large number of potential scatterers. A picture of the environment is given in Fig. 1. The SR employed is based on Novelda's X4M03 chip. The main parameters of the SR and of the measurement campaign are summarized in Tab. I. A total of 12 different spatial configurations (position and orientation) were used, 4 with the SR facing the center of the room as in a possible displacement for the SRN operation, and 8 where the SR is placed at center of the area facing 8 different directions. 
The configurations are indexed by a number representing the position and a subscript representing the orientation (see Fig. 2). The measurements at different configurations are analyzed separately and composed of $M=250$ frames and $N=1245$ samples per frame. The frames cover a range of approximately $8 \mathrm{~m}$.

\section{Statistical Characterization}

We now investigate the stationarity, correlation, and distribution of received signal samples $x(t)$ after clutter mitigation filtering. On the one hand, stationarity and correlation are properties of the process $x_{i}$, which depend on the discrete temporal index $i$. On the other hand, the process distribution is defined as the distribution of the $\mathrm{RV} \mathrm{x}_{i^{\star}}$, where $i^{\star}$ is a fixed time index with $i^{\star} \in \mathcal{N}$. In our case, stationarity and correlation are evaluated from the samples $\left\{x_{i}^{(j)}\right\}_{i \in \mathcal{N}}$ for each frame indexed by $j$, while samples distribution is evaluated from the samples $\left\{x_{i}^{(j)}\right\}_{j \in \mathcal{M}}$ for each sample indexed $i$.

As a first qualitative test, we use visual plots of the firstorder statistics (e.g., mean and variance) to evaluate the nonstationarity of the process by determining if such statistics depend on the sample index $i$. In particular, the sample mean is $\hat{\mu}_{i}=\sum_{j \in \mathcal{M}} x_{i}^{(j)} / M$, while the sample variance is $\hat{\sigma}_{i}^{2}=$ $\sum_{j \in \mathcal{M}}\left(x_{i}^{(j)}-\hat{\mu}_{i}\right)^{2} /(M-1)$. Indeed, if such dependency can be assessed by visual inspection, i.e., $\hat{\mu}_{i} \neq \hat{\mu}_{k}$ or $\hat{\sigma}_{i}^{2} \neq \hat{\sigma}_{k}^{2}$ for $i \neq k$ and $i, k \in \mathcal{N}$, the process $\mathrm{x}_{i}$ can be assumed nonstationary. Otherwise, if such dependency cannot be identified, quantitative tests are necessary to asses the process stationarity.

As a second quantitative test, we use the LBT for evaluating the correlation of the process $\mathrm{x}_{i}$. Assume $\mathrm{x}_{i}$ as stationary and denote the discrete autocorrelation function at time lag $k$ (i.e., sample index difference) as $\varrho_{k}$. Formally, the LBT tests the hypotheses

$$
\begin{aligned}
& \mathcal{H}_{0}: \quad \forall k \in \mathcal{N}_{\mathrm{L}}, \quad \varrho_{k}=0 \\
& \mathcal{H}_{1}: \quad \exists k \in \mathcal{N}_{\mathrm{L}}, \quad \varrho_{k} \neq 0
\end{aligned}
$$

where $\mathcal{N}_{\mathrm{L}}=\left\{1,2, \ldots, N_{\mathrm{L}}\right\}$, and $N_{\mathrm{L}}$ is the maximum time lag tested. In our case, we can apply the LBT for each frame index $j$ on the samples $\left\{x_{i}^{(j)}\right\}_{i \in \mathcal{N}}$. Consider a frame index $j$ and $\hat{\mu}_{i}=\hat{\mu}=0$, then the $\varrho_{k}$ can be estimated as $\hat{\varrho}_{k}^{(j)}=\hat{v}_{k}^{(j)} / \hat{v}_{0}^{(j)}$ where

$$
\hat{v}_{k}^{(j)}=\frac{1}{N} \sum_{h=1}^{N-k} x_{h}^{(j)} x_{h+k}^{(j)}
$$

is the estimate of the autocovariance function. The test statistic employed to reject the null hypothesis is based on the estimated autocorrelation function and is given by

$$
q^{(j)}=N(N+2) \sum_{k \in \mathcal{N}_{\mathrm{L}}} \frac{\left(\hat{\varrho}_{k}^{(j)}\right)^{2}}{N-k} .
$$

If the test statistic $q^{(j)}$ exceeds a certain critical value $\lambda_{\alpha}$, i.e. $q^{(j)}>\lambda_{\alpha}$, the hypothesis $\mathcal{H}_{0}$ is rejected with significance level $\alpha$ (i.e., the samples can be assumed correlated). Otherwise, the test fails to reject $\mathcal{H}_{0}$ and the samples are considered uncorrelated. Critical values for different significance levels $\alpha$ and sample sizes are available [33]. In the asymptotic regime $\left(N_{\mathrm{L}} \rightarrow+\infty\right)$ the test statistic $\mathrm{q}$ is distributed as a chi-square distribution with $N_{\mathrm{L}}$ degrees of freedom and the critical value $\lambda_{\alpha}$ is the $1-\alpha$ quantile of the distribution.

Various statistical tests can be employed for determining if the samples are well-modeled by a Gaussian distribution. In our case, we want to test for each sample index $i$ if the samples $\left\{x_{i}^{(j)}\right\}_{j \in \mathcal{M}}$ can be considered realizations of a Gaussian RV. Consider a fixed time index $i$, formally goodness-of-fit tests for Gaussian distributions verifies the hypothesis

$$
\begin{array}{lll}
\mathcal{H}_{0}: & \left\{x_{i}^{(j)}\right\}_{j \in \mathcal{M}}, & f_{\mathrm{x}_{i}}(x) \in \mathcal{F}\left(x ; \mu, \sigma^{2}\right) \\
\mathcal{H}_{1}: & \left\{x_{i}^{(j)}\right\}_{j \in \mathcal{M}}, & f_{\mathrm{x}_{i}}(x) \notin \mathcal{F}\left(x ; \mu, \sigma^{2}\right)
\end{array}
$$

where $\mathcal{F}\left(x ; \mu, \sigma^{2}\right)$ is a family of Gaussian distributions parametrized by mean $\mu$ and variance $\sigma^{2}$. ADT is employed to test Gaussianity since it exhibits a small probability of incurring in type-II error compared to other tests and it is able to detect small departures from the Gaussian distribution in the tails of the empirical distribution [33]. The ADT quantifies the square difference between the empirical distribution function defined as

$$
\tilde{F}_{i, M}(x)=\frac{\left|\left\{x_{i}^{(j)}, j \in \mathcal{M}: x_{i}^{(j)} \leq x\right\}\right|}{M}
$$

and the hypothesized CDF $F_{x_{i}}(x)$. In case of known mean and variance, the functional form of the test statistic is given by

$$
a_{i}=M \int_{-\infty}^{+\infty} \frac{\left(\tilde{F}_{i, M}(x)-F_{\mathrm{x}_{i}}(x)\right)^{2}}{F_{\mathrm{x}_{i}}(x)\left(1-F_{\mathrm{x}_{i}}(x)\right)} d F_{\mathrm{x}_{i}}(x)
$$

where the term at the denominator $F_{\mathrm{x}_{i}}(x)\left(1-F_{\mathrm{x}_{i}}(x)\right)$ can be seen as a weighting function assigning more weight to the difference in the distribution tails. Operatively, exploiting the property that a RV composed with its true CDF is uniformly distributed, the test statistic $a_{i}$ can be evaluated from the ordered samples $x_{i}^{(1)} \leq x_{i}^{(2)} \leq \ldots \leq x_{i}^{(M)}$ as

$$
a_{i}=-M-\frac{1}{M} \sum_{j \in \mathcal{M}}\left[(2 j-1) \ln \left(z_{i}^{(j)}\right)+\ln \left(z_{i}^{(M+1-j)}\right)\right]
$$

where $z_{i}^{(j)}=\tilde{F}_{i, M}\left(x_{i}^{(j)}\right)^{1}$. The hypothesis $\mathcal{H}_{0}$ is rejected at significance level $\alpha$, i.e. the samples are not considered Gaussian distributed if $a_{i}>\lambda_{\alpha}$. Similarly to the LBT, $\lambda_{\alpha}$ has been tabulated for different sample sizes and significance level $\alpha$ [33]. Differently from the LBT, the asymptotic distribution of the test statistic a has no closed form and the critical values have been obtained via Monte Carlo methods.

\section{RESUlts}

Consider the experimental setting described in Sec. II-C. Given a specific spatial configuration of the SR, we define $R_{\mathrm{C}}=N_{\mathrm{C}} / M$ as the ratio between the number of frames that

\footnotetext{
${ }^{1}$ If the mean and variance are estimated from the samples $\left\{x_{i}^{(j)}\right\}_{j \in \mathcal{M}}$, a correction factor needs to be multiplied to the test statistic.
} 


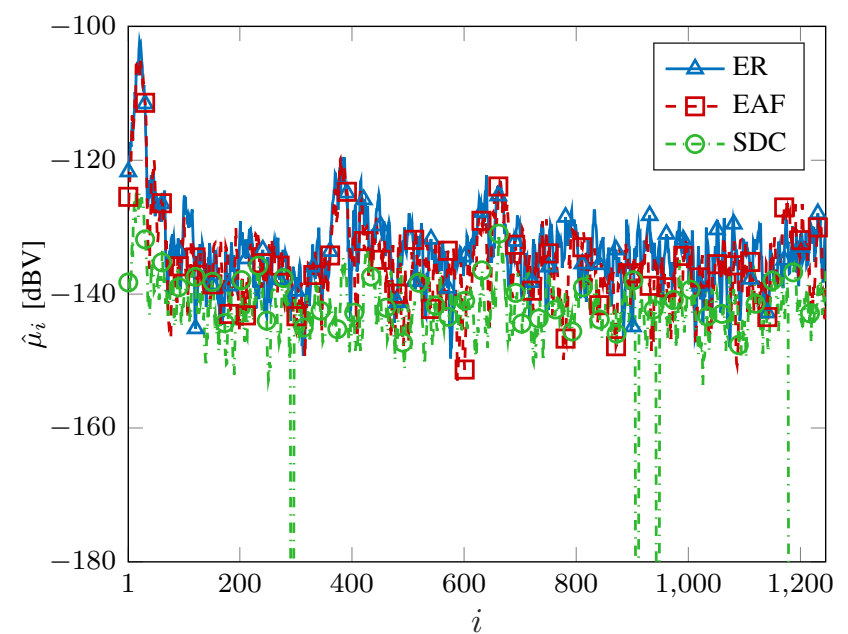

Fig. 3. Sample mean for the configuration $5_{\mathrm{E}}$ with the three different clutter mitigation filters employed: ER, EAF, and SDC.

exhibit correlation $N_{\mathrm{C}}$, assessed via LBT, and the total number of frame $M$ in each configuration. Similarly, given a specific configuration we define $R_{\mathrm{G}}=N_{\mathrm{G}} / M$ as the ratio between the number of sample that can be considered Gaussian distributed $N_{\mathrm{G}}$, assessed via ADT, and the total number of samples $N$ in each configuration. Both LBT and ADT are conducted at significance level $\alpha=0.05$, with $N_{\mathrm{L}}=20$ for the LBT. The weighting parameter for the EAF is $\beta=0.8$.

Fig. 3 shows the sample mean for the configuration $5_{\mathrm{E}}$ as a function of the sample index $i$ for the different clutter mitigation filters considered. It can be observed that the mean estimate exhibits great variability from sample to sample. In particular, it can be identified a peak of approximatively -100 $\mathrm{dB}$ in the first 50 samples for all the mitigation filters. This can be attributed to the direct path between the transmitter and receiver antennas. Moreover, other two peaks of approximatively $-120 \mathrm{~dB}$ and width of several samples can be identified at $i \approx 400$ and $i \approx 600$. These can be attributed to large scatterers, such as the table and the wall. At the same sample indices, similar peaks can be recognized in Fig. 4, where it is showed the sample variance for the same configuration as a function of the sample index $i$. Similar trends can be observed for the mean and the variance in the other configurations recorded, where peaks can be identified in correspondence of large scattered echoes. Furthermore, these peaks are present regardless of the mitigation filter considered. Notice that, the presence of such peaks violate the definition of stationarity and their width is attributable to the characteristics of the UWB signals. In fact, due to the fine spatial resolution provided by UWB signals, the mitigated echoes from static scatterers span multiple sample indices.

Tab. II shows $R_{\mathrm{C}}$ for the configurations considered and different clutter mitigation filters. It can be noticed that the percentage of frames exhibiting correlation exceeds the $80 \%$ for all the configurations and mitigation filters. It can also be noticed that the filtered samples obtained with the ER exhibit the larger percentage (greater than $90 \%$ ) of correlated frames,

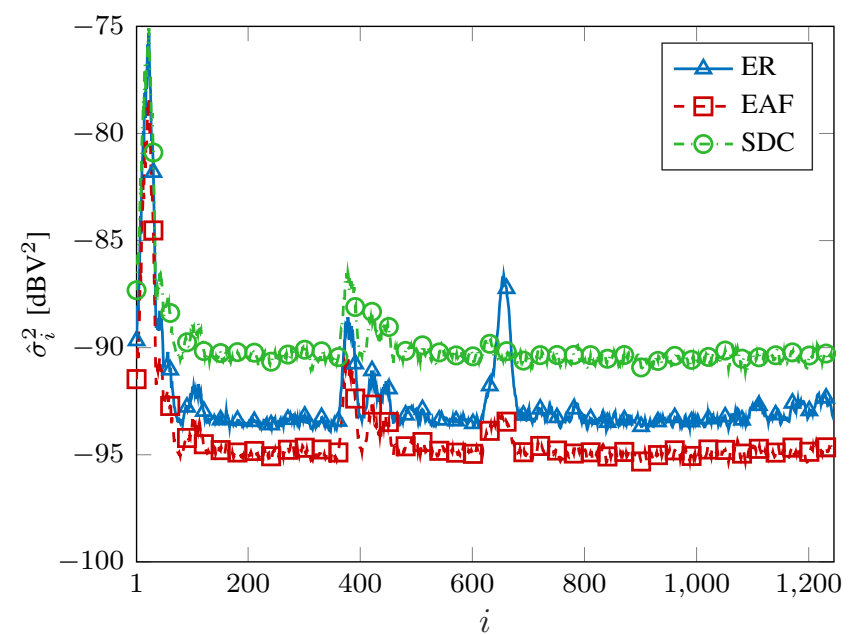

Fig. 4. Sample variance for the configuration $5_{\mathrm{E}}$ with the three different clutter mitigation filters employed: ER, EAF, and SDC.

while the lowest is obtained employing the SDC. This can be attributed to the fact that the ER filter does not affect the statistical properties of the input process, being the output process a simple location shift of the input process. On the other hand, the SDC mitigates the clutter and increases the power of the AWGN which is assumed uncorrelated.

Tab. III shows $R_{\mathrm{G}}$ for the configuration considered and different clutter mitigation filters. It can be noticed that the percentage of samples well modeled by a Gaussian distribution exceeds the $90 \%$ in the majority of the configurations. In particular, for EAF and SDC the $R_{\mathrm{G}}$ is almost $95 \%$ for all the configuration. In the case of ER, the values of $R_{\mathrm{G}}$ is slightly inferior, with $R_{\mathrm{G}}$ greater than $90 \%$ in all location, except for $2_{\mathrm{N}}$ where $R_{\mathrm{G}} \approx 82 \%$. This can be attributed to minor variation of the environment between the formation of the ER response and the actual measurement.

Given the results, the process describing the sample amplitudes after clutter mitigation filtering can be considered Gaussian distributed. Moreover, this process can be assumed non-stationary in the first-order statistics and exhibits correlation. The non-stationarity can be attributed to the large number of potential scatterers in indoor environments and the characteristics of the UWB signals, in particular to their fine spatial resolution. These are important properties that must be taken into account for the derivation of performance benchmarks and the design of robust inference algorithms.

\section{FinAl REMARK}

This paper has proposed an approach to characterize the static clutter in indoor environments for sensor radar networks employing ultra-wideband (UWB) signals. In particular, the clutter-plus-noise at the output of clutter mitigation filters is modeled as a stochastic process and its statistical properties have been derived based on statistical tests applied on real measurements. Results shows that the sample amplitudes can be considered as a non-stationary correlated Gaussian process, where the non-stationarity and correlation are attributable to 
TABLE II

RATE OF CORRELATED FRAMES FOR DIFFERENT CONFIGURATIONS AND CLUTTER MITIGATION FILTERING.

\begin{tabular}{|c|c|c|c|}
\hline \multirow{2}{*}{ Configuration } & \multicolumn{3}{|c|}{ Rate of Correlated Frames, $R_{\mathrm{C}}$} \\
\cline { 2 - 4 } & ER & EAF & SDC \\
\hline $1_{\mathrm{E}}$ & 0.92 & 0.87 & 0.80 \\
$2_{\mathrm{N}}$ & 0.99 & 0.88 & 0.85 \\
$3_{\mathrm{W}}$ & 0.95 & 0.88 & 0.86 \\
$4_{\mathrm{S}}$ & 0.99 & 0.92 & 0.81 \\
$5_{\mathrm{E}}$ & 0.95 & 0.85 & 0.80 \\
$5_{\mathrm{NE}}$ & 0.94 & 0.86 & 0.83 \\
$5_{\mathrm{N}}$ & 0.99 & 0.88 & 0.84 \\
$5_{\mathrm{NW}}$ & 0.96 & 0.88 & 0.82 \\
$5_{\mathrm{W}}$ & 0.92 & 0.85 & 0.80 \\
$5_{\mathrm{SW}}$ & 0.96 & 0.88 & 0.85 \\
$5_{\mathrm{S}}$ & 0.95 & 0.87 & 0.81 \\
$5_{\mathrm{SE}}$ & 0.98 & 0.85 & 0.80 \\
\hline
\end{tabular}

the indoor environment and UWB signal characteristics. The knowledge of such model represents a first step into the clutter characterization problem in a more general condition and it is an essential element for the derivation of performance benchmarks and the design of tracking algorithms.

\section{REFERENCES}

[1] O. Andrisano et al., "The need of multidisciplinary approaches and engineering tools for the development and implementation of the smart city paradigm," Proc. IEEE, vol. 106, no. 4, pp. 738-760, Apr. 2018.

[2] R. S. Thoma et al., "Cooperative passive coherent location: A promising 5G service to support road safety," IEEE Commun. Mag., vol. 57, no. 9, pp. 86-92, Sep. 2019.

[3] S. M. George et al., "Distressnet: A wireless Ad Hoc and sensor network architecture for situation management in disaster response," IEEE Commun. Mag., vol. 48, no. 3, pp. 128-136, Mar. 2010.

[4] M. Chiani, A. Giorgetti, and E. Paolini, "Sensor radar for object tracking," Proc. IEEE, vol. 106, no. 6, pp. 1022-1041, Jun. 2018

[5] A. Conti, S. Mazuelas, S. Bartoletti, W. C. Lindsey, and M. Z. Win, "Soft information for localization-of-things," Proc. IEEE, vol. 107, no. 11, pp. 2240 - 2264, Nov. 2019

[6] J. Salmi and A. F. Molisch, "Propagation parameter estimation, modeling and measurements for ultrawideband MIMO radar," IEEE Trans. Antennas Propag., vol. 59, no. 11, pp. 4257-4267, Nov. 2011.

[7] R. Niu, R. S. Blum, P. K. Varshney, and A. L. Drozd, "Target localization and tracking in noncoherent multiple-input multiple-output radar systems," IEEE Trans. Aerosp. Electron. Syst., vol. 48, no. 2, pp. 14661489, Apr. 2012.

[8] F. Morselli, S. Bartoletti, S. Mazuelas, M. Z. Win, and A. Conti, "Crowdcentric counting via unsupervised learning," in 2019 IEEE Int. Conf. on Communications Workshops (ICC Workshops). IEEE, May 2019.

[9] M. I. Skolnik, Radar Handbook, 3rd ed. New York, NY 10020: McGraw-Hill, 1970.

[10] S. Bartoletti, A. Giorgetti, M. Z. Win, and A. Conti, "Blind selection of representative observations for sensor radar networks," IEEE Trans. Veh. Technol., vol. 64, no. 4, pp. 1388-1400, Apr. 2015.

[11] M. Z. Win, Y. Shen, and W. Dai, "A theoretical foundation of network localization and navigation," Proc. IEEE, vol. 106, no. 7, pp. 1136-1165, Jul. 2018.

[12] E. Conte and A. D. Maio, "Mitigation techniques for non-Gaussian sea clutter," IEEE J. Ocean. Eng., vol. 29, no. 2, pp. 284-302, Apr. 2004.

[13] A. Balleri, A. Nehorai, and J. Wang, "Maximum likelihood estimation for compound-Gaussian clutter with inverse gamma texture," IEEE Trans. Aerosp. Electron. Syst., vol. 43, no. 2, pp. 775-779, Apr. 2007.

[14] F. Meyer et al., "Message passing algorithms for scalable multitarget tracking," Proc. IEEE, vol. 106, no. 2, pp. 221-259, Feb. 2018.

[15] B. Yazici and G. Xie, "Wideband extended range-Doppler imaging and waveform design in the presence of clutter and noise," IEEE Trans. Inf. Theory, vol. 52, no. 10, pp. 4563-4580, Oct. 2006.
TABLE III

RATE OF GAUSSIAN SAMPLES FOR DIFFERENT CONFIGURATIONS AND CLUTTER MITIGATION FILTERING.

\begin{tabular}{|c|c|c|c|}
\hline \multirow{2}{*}{ Configuration } & \multicolumn{3}{|c|}{ Rate of Gaussian Samples, $R_{\mathrm{G}}$} \\
\cline { 2 - 4 } & ER & EAF & SDC \\
\hline $1_{\mathrm{E}}$ & 0.94 & 0.96 & 0.96 \\
$2_{\mathrm{N}}$ & 0.82 & 0.94 & 0.96 \\
$3_{\mathrm{W}}$ & 0.91 & 0.94 & 0.96 \\
$4_{\mathrm{S}}$ & 0.95 & 0.96 & 0.96 \\
$5_{\mathrm{E}}$ & 0.93 & 0.95 & 0.96 \\
$5_{\mathrm{NE}}$ & 0.93 & 0.95 & 0.96 \\
$5_{\mathrm{N}}$ & 0.93 & 0.96 & 0.95 \\
$5_{\mathrm{NW}}$ & 0.92 & 0.95 & 0.95 \\
$5_{\mathrm{W}}$ & 0.94 & 0.95 & 0.96 \\
$5_{\mathrm{SW}}$ & 0.94 & 0.95 & 0.96 \\
$5_{\mathrm{S}}$ & 0.94 & 0.94 & 0.96 \\
$5_{\mathrm{SE}}$ & 0.91 & 0.96 & 0.95 \\
\hline
\end{tabular}

[16] E. Paolini, A. Giorgetti, M. Chiani, R. Minutolo, and M. Montanari, "Localization capability of cooperative anti-intruder radar systems," EURASIP J. Adv. Signal Processing, vol. 2008, pp. 1-14, Apr. 2008.

[17] V. Anastassopoulos, G. Lampropoulos, A. Drosopoulos, and N. Rey, "High resolution radar clutter statistics," IEEE Trans. Aerosp. Electron. Syst., vol. 35, no. 1, pp. 43-60, Jan. 1999.

[18] S. Haykin, R. Bakker, and B. Currie, "Uncovering nonlinear dynamicsthe case study of sea clutter," Proc. IEEE, vol. 90 , no. 5, pp. 860-881, May 2002.

[19] M. Greco, F. Bordoni, and F. Gini, "X-band sea-clutter nonstationarity: Influence of long waves," IEEE J. Ocean. Eng., vol. 29, no. 2, pp. 269283, Apr. 2004

[20] F. Gini and A. Farina, "Vector subspace detection in compound-Gaussian clutter. Part I: Survey and new results," IEEE Trans. Aerosp. Electron. Syst., vol. 38, no. 4, pp. 1295-1311, Oct. 2002.

[21] F. Gini, A. Farina, and M. Montanari, "Vector subspace detection in compound-Gaussian clutter. Part II: Performance analysis," IEEE Trans. Aerosp. Electron. Syst., vol. 38, no. 4, pp. 1312-1323, Oct. 2002.

[22] Y. Shen and M. Z. Win, "Fundamental limits of wideband localization Part I: A general framework," IEEE Trans. Inf. Theory, vol. 56, no. 10, pp. 4956-4980, Oct. 2010.

[23] S. Gezici, Z. Tian, G. B. Giannakis, H. Kobayashi, A. F. Molisch, H. V. Poor, and Z. Sahinoglu, "Localization via ultra-wideband radios: A look at positioning aspects for future sensor networks," IEEE Signal Process. Mag., vol. 22, pp. 70-84, Jul. 2005.

[24] D. Dardari, A. Conti, U. J. Ferner, A. Giorgetti, and M. Z. Win, "Ranging with ultrawide bandwidth signals in multipath environments," Proc. IEEE, vol. 97, no. 2, pp. 404-426, Feb. 2009.

[25] S. Bartoletti, W. Dai, A. Conti, and M. Z. Win, "A mathematical model for wideband ranging," IEEE J. Sel. Topics Signal Process., vol. 9, no. 2, pp. 216-228, Mar. 2015.

[26] A. Conti, M. Guerra, D. Dardari, N. Decarli, and M. Z. Win, "Network experimentation for cooperative localization," IEEE J. Sel. Areas Commun., vol. 30, no. 2, pp. 467-475, Feb. 2012.

[27] S. Bartoletti, A. Conti, W. Dai, and M. Z. Win, "Threshold profiling for wideband ranging," IEEE Signal Process. Lett., vol. 25, no. 6, pp. 873-877, Jun. 2018.

[28] A. Lazaro, D. Girbau, and R. Villarino, "Techniques for clutter suppression in the presence of body movements during the detection of respiratory activity through UWB radars," Sensors, vol. 14, no. 2, pp. 2595-2618, Feb. 2014.

[29] G. M. Ljung and G. E. P. Box, "On a measure of lack of fit in time series models," Biometrika, vol. 65, no. 2, pp. 297-303, Aug. 1978.

[30] T. W. Anderson and D. A. Darling, "A test of goodness of fit," J. Amer. Stat. Assoc., vol. 49, no. 268, pp. 765-769, Dec. 1954.

[31] M. Z. Win and R. A. Scholtz, "Impulse radio: How it works," IEEE Commun. Lett., vol. 2, no. 2, pp. 36-38, Feb. 1998.

[32] H. L. V. Trees, Detection, Estimation, and Modulation Theory, 1st ed. New York, NY 10158-0012: John Wiley \& Sons, Inc., 1968.

[33] R. B. D'Agostino and M. A. Stephens, Goodness-of-Fit-Techniques. Taylor \& Francis Inc, 1986. 